(C) 2017. This manuscript version is made available under the CC-BY-NC-ND 4.0 license http://creativecommons.org/licenses/by-nc-nd/4.0/

\title{
Nanofabrication of Phase-Shifted Bragg Gratings on the End Facet of Multimode Fiber towards Development of Optical Filters and Sensors
}

\author{
E. E. Gallego', J. Ascorbe ${ }^{2}$, I. Del Villar ${ }^{2,3}$, J. M. Corres ${ }^{2,3}$ and I.R. Matias ${ }^{2,3}$ \\ ${ }^{1}$ Department of Telecommunications and Electronics, Pinar del Río University, Pinar del Río CP \\ 20100, Cuba \\ ${ }^{2}$ Department of Electrical and Electronic Engineering, Public University of Navarra, 31006 \\ Pamplona, Spain \\ ${ }^{3}$ Institute of Smart Cities, Public University of Navarra, Pamplona 31006, Spain \\ Corresponding author: ignacio.delvillar@unavarra.es
}

\begin{abstract}
This work describes the process of nanofabrication of phase-shifted Bragg gratings on the end facet of a multimode optical fiber with a pulsed DC sputtering system based on a single target. Several structures have been explored as a function of parameters such as the number of layers or the phase-shift. The experimental results, corroborated with simulations based on plane-wave propagation in a stack of homogeneous layers, indicate that the phase-shift can be controlled with a high degree of accuracy. The device could be used both in communications, as a filter, or in the sensors domain. As an example of application, a humidity sensor with wavelength shifts of $12 \mathrm{~nm}$ in the range of 30 to $90 \%$ relative humidity (200 pm/\% relative humidity) is presented.
\end{abstract}


Keywords: optical fiber components, Bragg gratings, thin-films, optical fiber sensors, band-pass filters, fiber DC sputtering.

\section{INTRODUCTION}

The deposition of a stack of high and low refractive index materials on different substrates, such as silicon wafers, quartz, BK7 glass, or optical fiber, has been explored in several publications with the aim of developing optical filters and sensors [1-10].

In this work the substrate will be the end facet of an optical fiber, which allows obtaining a device that includes many interesting properties. On the one hand, optical fiber is immune against electromagnetic interferences, presents small diameter and high bandwidth, and is capable of multiplexing several signals. On the other hand, though some authors consider the deposition of high and low refractive index layers as a multilayer stack [1,3-4,6-8] or a micrograting [2], others use more subtle terms such as Bragg stacks [5], or simply Bragg gratings [10]. This last term is more adequate in the work presented here because focus is centered on the monitorization of bands generated in the reflected spectrum according to the Bragg-Snell equation for normal incidence [5]:

$$
m \lambda=2\left(n_{H} h_{H}+n_{L} h_{L}\right)
$$

where $m$ is the diffraction order and $n_{H}, n_{L}, h_{H}$, and $h_{L}$ are the refractive indices and thicknesses of the high- $(\mathrm{H})$ and low- $(\mathrm{L})$ refractive index materials respectively. All these variables increase the potential of optical fiber and allow controlling the shape of the optical spectrum, as it will be shown later, which can be used both for the design of optical filters and sensors. Indeed, the direct contact of the Bragg grating with the outer medium, combined with the small size of the micrograting makes this type of structure a good candidate for a small-size sensing probe. 
Regarding the techniques used for nanodeposition of each layer in the Bragg grating, there are some works focused on layer-by-layer (LbL) self-assembly [2,11]. However, even though much progress has been done in the last years in terms of increasing the deposition speed [12], sputtering is the most adequate technique for this type of structures due to the fine control in the nanocoating thickness it offers, by applying a specific combination of parameters such as power, current, vacuum pressure or gas flow rate $[13,14]$. This makes sputtering a useful tool for deposition of nanocoatings on optical fibers [15-17].

In a recent work it has been proved that it is possible to develop a Bragg grating on the end facet of an optical fiber by DC sputtering technique with a single target, which increases the simplicity of the nanodeposition process [10]. In this work, the possibility of including a variation of the thickness in one of the layers of the grating is explored. This type of structure is considered in the literature as a one-dimensional photonic band gap structure (PBG) with a defect [11]. However, it is more usual to use the term phase-shifted grating [18-21]. Consequently, this notation will be used henceforward. As an example of application of this type of device, a humidity sensor will be shown, which opens the possibility to develop other sensing applications where the defect layer is even composed of another material that is sensitive to other parameters. In section 2 the experimental setup and the methods used for fabricating and simulating the Bragg gratings will be explained. In section 3 the experimental results are detailed. Finally, some conclusions are included in section 4.

\section{METHODS AND MATERIALS}

This section describes the process of generation of a grating and the possibility to generate a phase-shift in the middle of the grating, as well as the numerical analysis used to simulate the structure. 


\section{Generation of gratings with pulsed DC sputtering}

Figure 1 shows the experimental setup used in the construction process of the gratings. In this set-up, a white light source ANDO and a HR4000 spectrometer were used to monitor, in a reflection configuration, the deposition process on the fiber end facet with SpectraSuite software. A pulsed DC sputtering equipment (Nadetech Innovations, Ltd.) with a single target of $\mathrm{SnO}_{2}$ was used for the deposition. In order to control the generation of high and low refractive index layers, the gas injection into the vacuum chamber was automated with a LabView controlled gas panel, which allowed modifying the properties of the material deposited at any moment.

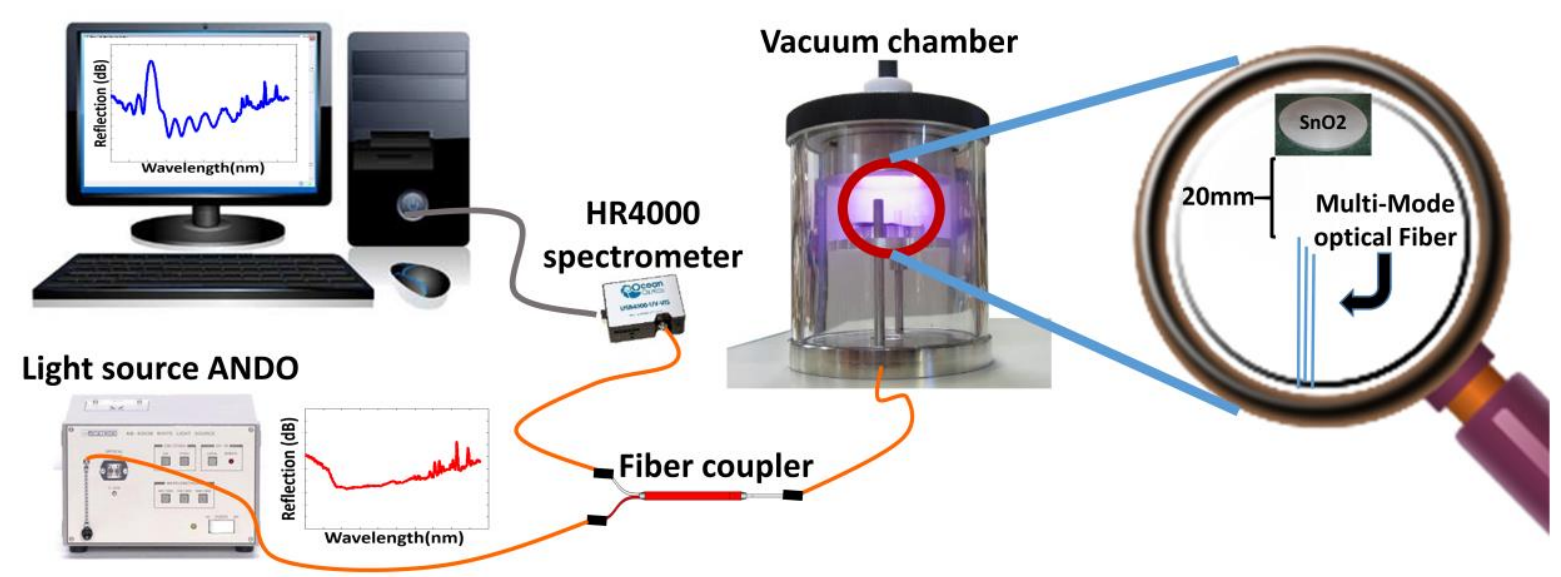

Fig. 1. Experimental setup used to monitor the pulsed DC sputtering process.

Figure 2 shows a schematic representation of a 7 period grating deposited on the end facet of a multi-mode optical fiber (core and cladding diameter 62.5/125 $\mu \mathrm{m}$ ) from Telnet Redes Inteligentes Inc. (Zaragoza, Spain). 


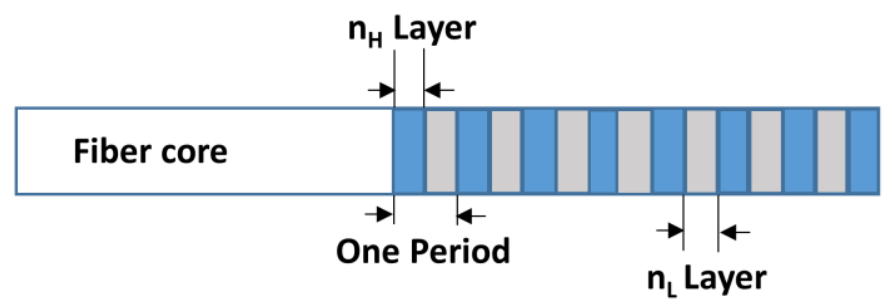

Fig. 2. Simple Bragg grating on the end facet of an optical fiber.

Successive deposition of the same material, $\mathrm{SnO}_{2}$, in two different conditions permitted to obtain high refractive index and low refractive index layers. The conditions for both materials are listed in Table I. The parameter that leads to the refractive index change is the gas combination (i.e. oxygen-argon for a high refractive index and only argon for a low refractive index layer). In this way, a refractive index contrast between layers created in oxygen-argon atmosphere and layers created only in argon atmosphere is obtained.

TABLE I

CONDITIONS FOR DC SPUTTERING DEPOSITION PROCESS

\section{Condition $\quad$ Value/description}

Deposited material

Deposition time per layer

Gas combination to achieve refractive index variation per layer

Pressure in vacuum chamber

Current intensity

Distance from the fiber end facet tip to the cathode
$\mathrm{SnO}_{2}$

30 seconds

Oxygen-Argon $\left(\mathrm{n}_{\mathrm{L}}\right)$ or only Argon $\left(\mathrm{n}_{\mathrm{H}}\right)$

$2 \times 10^{-2}$ to $3 \times 10^{-2}$ mbar

$0.21 \mathrm{~A}$

$18-24 \mathrm{~mm}$

The first layer of the period in the depositions process for all cases was an $n_{H}$ layer (high refractive index), and the second one was an $\mathrm{n}_{\mathrm{L}}$ layer (low refractive index).

Henceforward, we will refer to the set of two consecutive high and low index depositions, as a period. 
As an average value, the thickness of each layer was approximately of $80 \mathrm{~nm}$ with the current deposition time per layer (Table I). This thickness depends on the distance between the fiber tip and the cathode.

\section{Phase-shifted gratings}

Figure 3 shows two different examples of phase-shifted gratings: a $\pi$-phase-shifted grating and a $3 \pi$-phase-shifted grating. In terms of defects, a $\pi$-phase-shifted grating is equivalent to a defect of two layers or one period, whereas a $3 \pi$-phase-shifted grating is equivalent to a defect of four layers or two periods.

a)
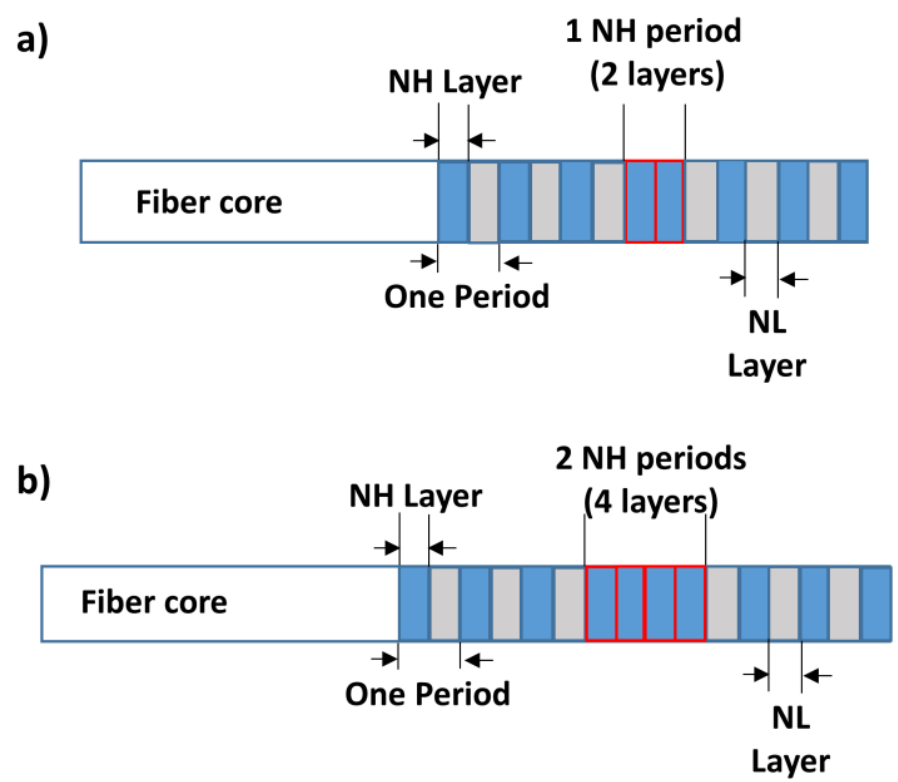

Figure 3: 6 period Bragg gratings: (a) with $\pi$-phase-shift, (b) with $3 \pi$-phase-shift.

For gratings with defects, the structure of the gratings before the defect is the same as the grating in the Figure 2, followed by one $\mathrm{nH}$ defect of a certain thickness, and starting the layers with $\mathrm{nL}$ after the defect. 
A $\pi$-phase-shift that allows generating a resonance in the center of the reflection band of the fiber grating without defect $[5,19]$. This phenomenon is periodic and is repeated with odd multiples of $\pi$. Consequently, the cases presented in Fig. 3, $\pi$-phase-shifted grating and $3 \pi$-phase-shifted grating, should lead to similar results.

In order to prove this idea, a simulation in Figure 4 shows the influence of the thickness in the defect layer (the simulation method is explained in the following subsection). In all cases the grating period was $160 \mathrm{~nm}$ and the duty cycle was $50 \%$ (i.e. $80 \mathrm{~nm}$ ).
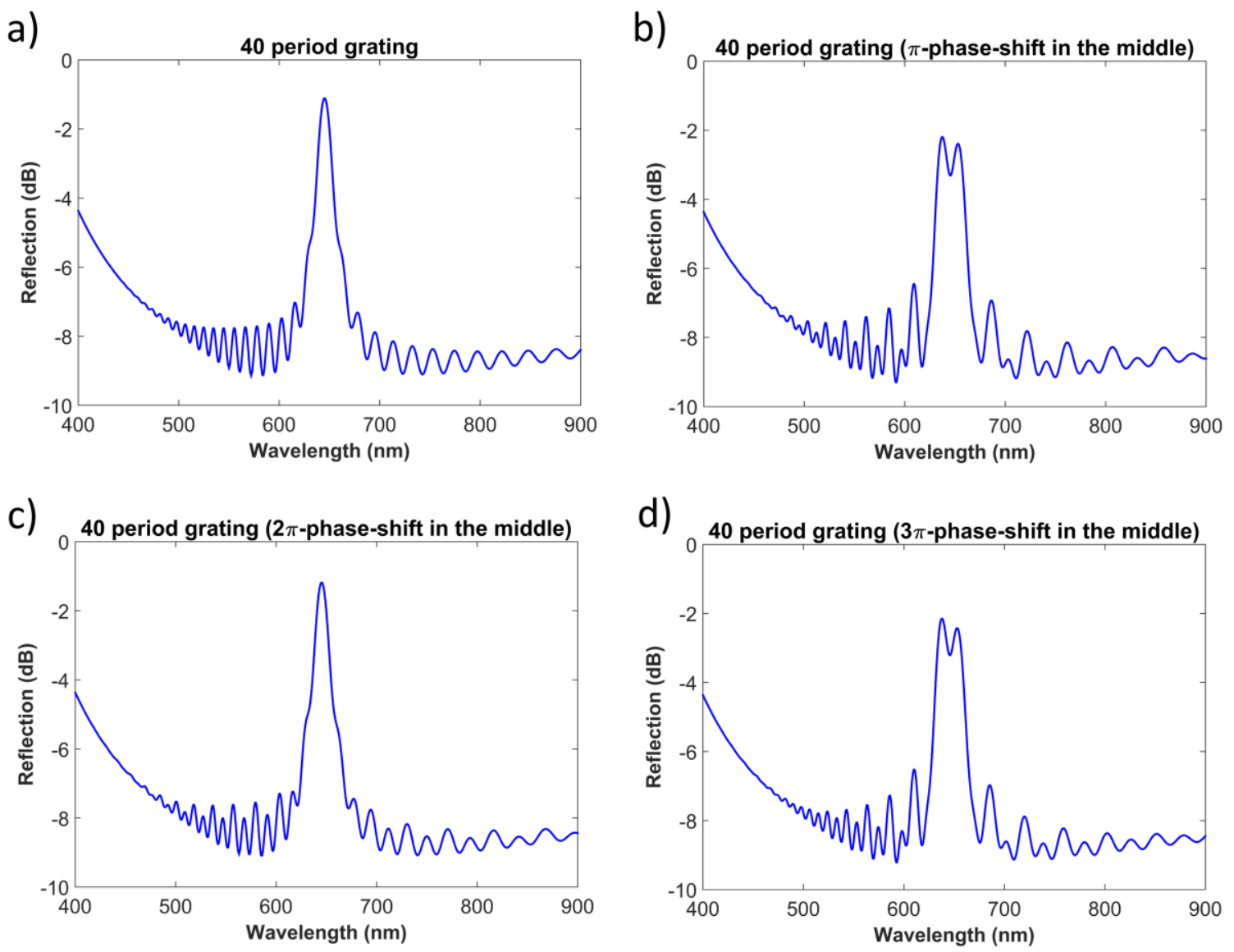

Fig. 4: Reflection spectrum for a grating of 40 periods with a phase-shift in the middle. Four different case were analyzed: (a) no phase-shift; (b) $\pi$-phase-shift; (c) $2 \pi$-phase-shift; (d) $3 \pi$-phaseshift. Visualization 1 shows the evolution of the optical spectrum as a function of thickness in the defect layer. 
With a defect of $160 \mathrm{~nm}$ ( $\pi$-phase-shift) a dip in the middle of the reflection band is created. The dip disappears if the defect thickness is $240 \mathrm{~nm}$ ( $2 \pi$-phase-shift), and the dip is again visible with a defect of $320 \mathrm{~nm}$ ( $3 \pi$-phase-shift). The complete evolution of the optical spectrum as a function of the defect thickness can be observed in Visualization 1.

The number of periods was 40 , as a trade-off value that allowed observing the reflection band with a dip in the middle for $\pi$ and $3 \pi$-phase-shift cases. If the number of periods is increased, the dip in the middle is not visible, whereas if the number of periods is reduced, the reflection band does not prevail over the lateral bands. This idea will be proved in section III.

\section{Simulation method and parameters}

The simulations in Fig. 4 and in the rest of the article have been performed with Matlab®, following a numerical method based on plane wave propagation in a one-dimensional stack of layers of different refractive indices [10,11]. Fused silica is the material of the multimode optical

fiber. Consequently the Sellmeier equation was used to calculate the refractive index as a function of wavelength:

$$
n^{2}(\omega)=1+\sum_{j=1}^{m} \frac{B_{j} \omega_{j}^{2}}{\omega_{j}^{2}-\omega^{2}}
$$

with parameters: $\quad B_{1}=0.691663, \quad B_{2}=0.4079426, \quad B_{3}=0.8974794, \quad \lambda_{1}=0.0684043 \quad \mu m, \quad \lambda_{2}=$ 0.1162414 , and $\lambda_{3}=9.896161$, where $\lambda_{\mathrm{j}}=2 \pi \mathrm{c} / \omega \mathrm{j}$ and $\mathrm{c}$ is the speed of light in vacuum [22].

Regarding the grating, an ellipsometer UVISEL with spectral range 0.6-6.5 eV (190 - $2100 \mathrm{~nm})$, an angle of incidence $70^{\circ}$, an spot size $1 \mathrm{~mm}$ and Software DeltaPsi2TM (from Horiba Scientific Thin Film Division) was used to calculate the refractive index of $\mathrm{SnO}_{2}$ as a function of wavelength (see Fig. 5). 


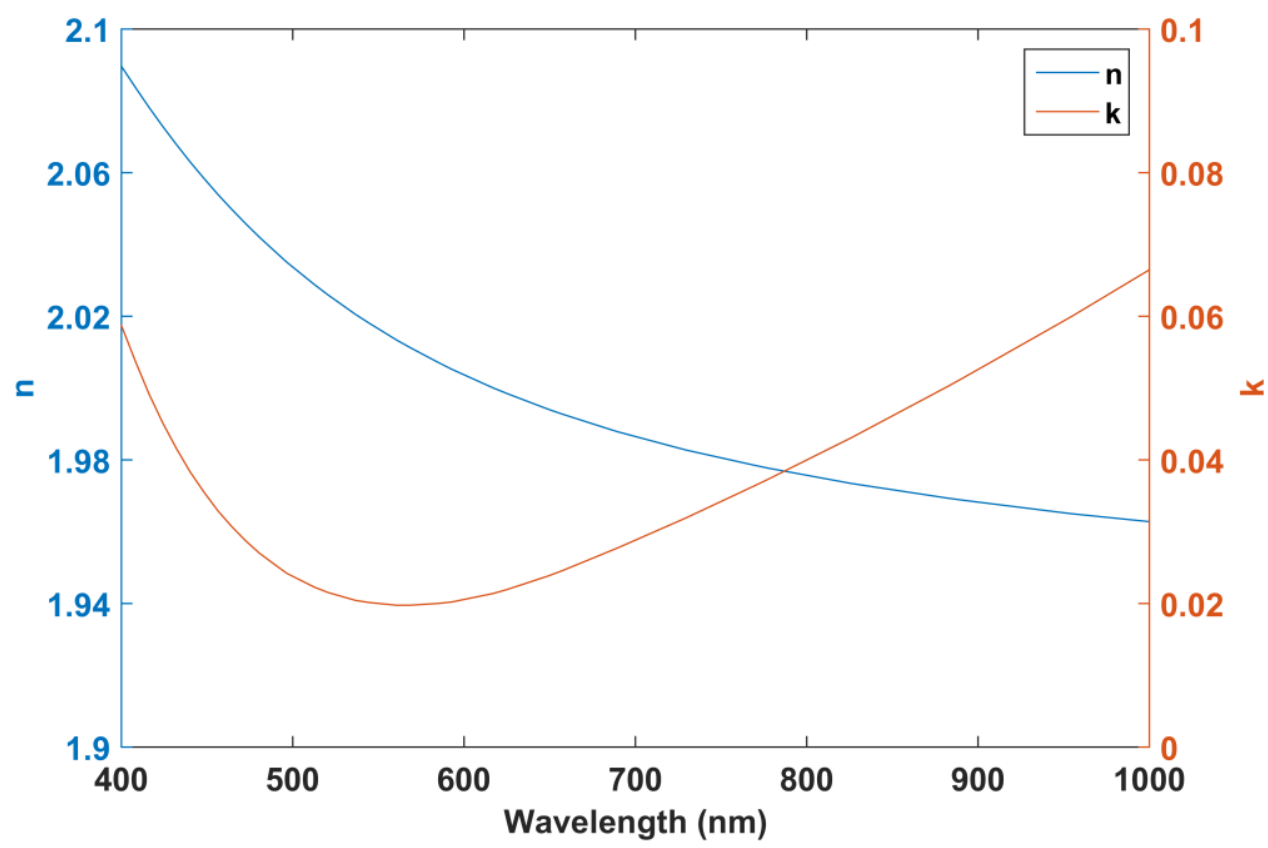

Fig. 5. Complex refractive index (n, k) dispersion curves of $\mathrm{SnO}_{2}$ coated silicon wafer.

In addition, according to the results obtained in simulations and experiments, the average contrast between the high and low refractive index layers is $2 \%$, which agrees with the results obtained in [10]. This parameterization was used for the simulations.

\section{RESULTS AND DISCUSSION}

\section{Fabrication of Bragg gratings with different parameters}

Figure 6 shows the reflection spectrum of a grating of 20 periods and the same structure with a $\pi$ phase-shifted grating. A lower period than in section 2 was selected in order to enhance the visibility of the phase-shift band. Thanks to this, the dip in the middle of Figure 6(b) is evident. In addition, the evolution of the spectra during the fabrication of the structures can be observed in Visualizations 2 and 3. 

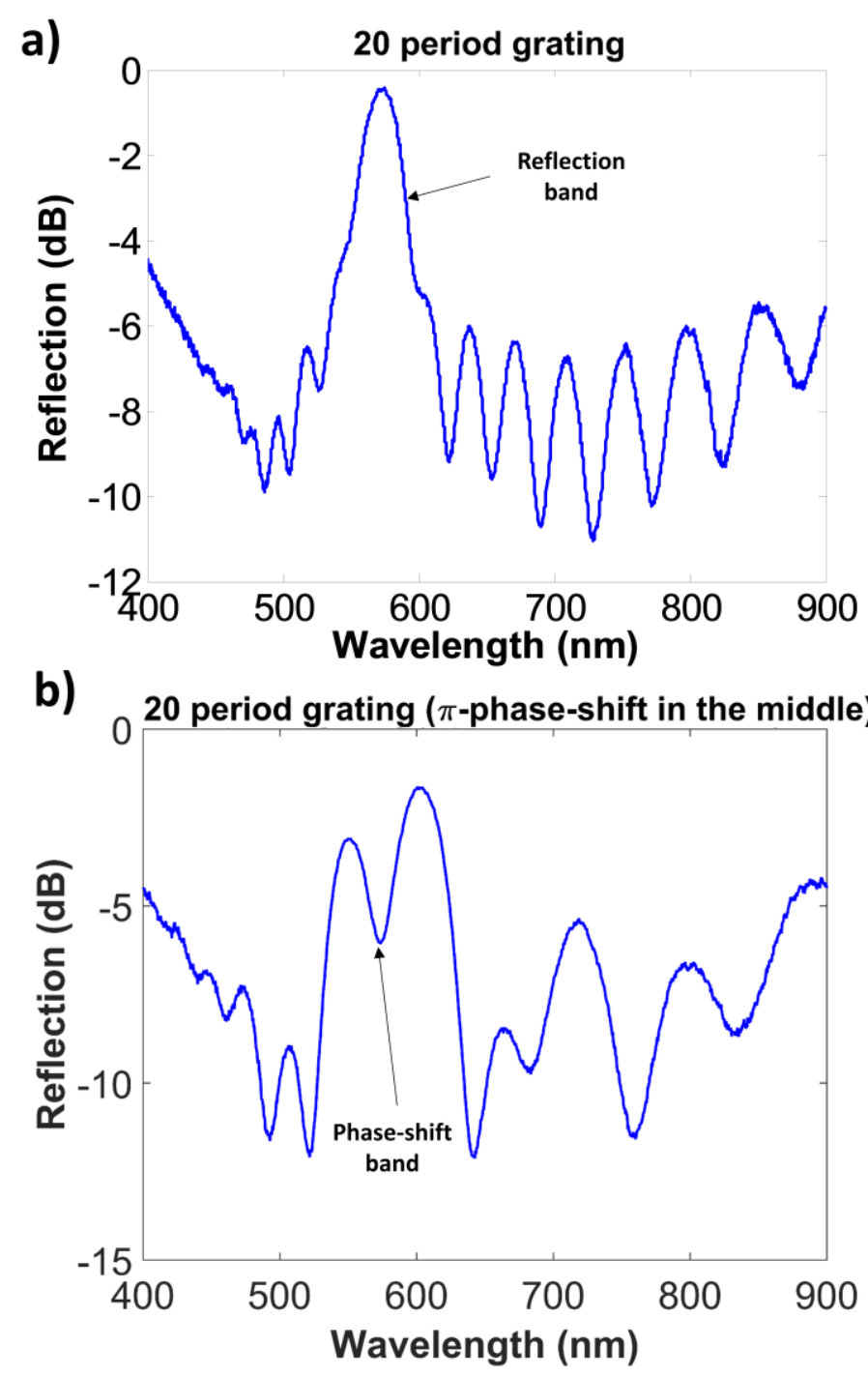

Fig. 6. Spectrum of (a) 20 period grating (b) 20 period grating with a $\pi$-phase-shift in the middle.

Henceforward, focus will be centered on phase-shifted structures. First, the structure described in Figure 3(b) was fabricated on three fiber tips positioned at different distance from the target of the sputtering machine: 20, 22 and $24 \mathrm{~mm}$ respectively. In Figure 7(a) the corresponding spectra are shown. A wavelength shift to the red is observed as the fiber is positioned closer to the target. This effect is due to the thicker grating is generated, which is corroborated with the theoretical results presented in Figure 7(b) for three different thickness values for the grating layers: 86, 89 and $92 \mathrm{~nm}$. 

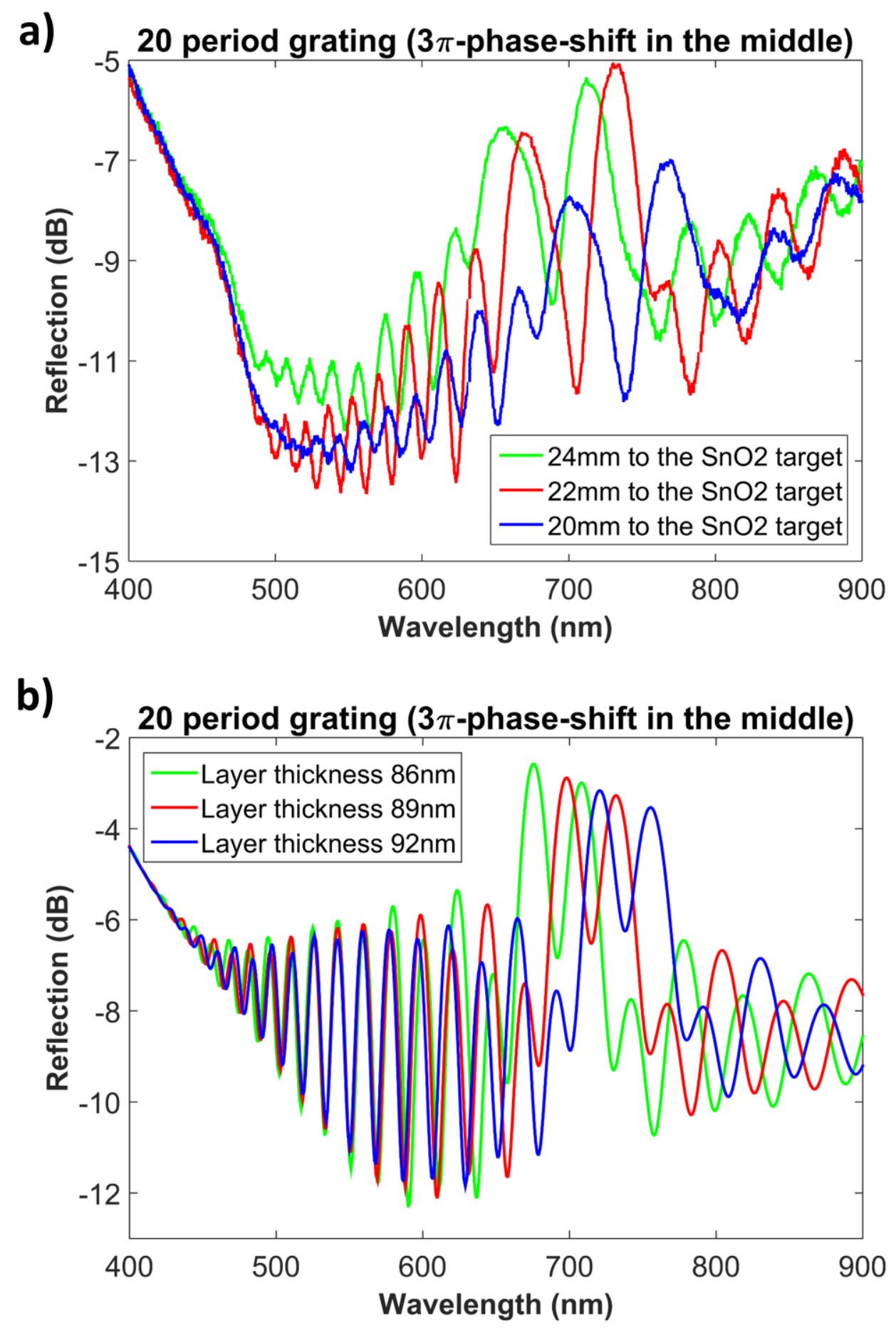

Fig. 7. Effect of the variation of the layers thickness for a 20 period grating with $3 \pi$-phase-shift in the middle: a) Experimentally fabrication, b) Simulation. 
The effect of layers thickness variation can be observed in Visualization 4. Moreover, a silicon wafer was deposited in parallel with the gratings and the section was analyzed in a field emission scanning electron microscope (SEM) UltraPlus, from Carl Zeiss Inc with an in-lens detector at $3 \mathrm{kV}$ and an aperture diameter of $30 \mu \mathrm{m}$ (see Fig. 8).

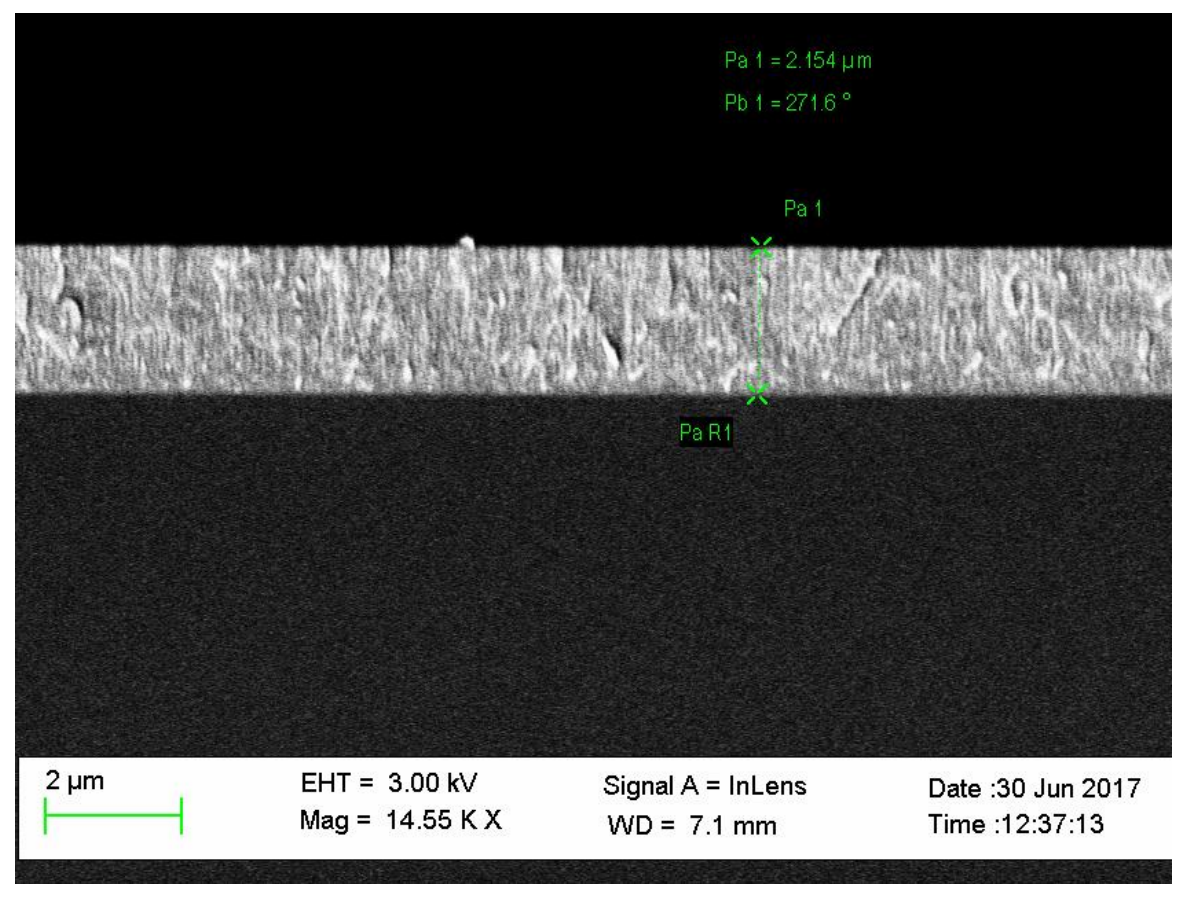

Fig. 8. Scanning electron microscope (SEM) of $\mathrm{SnO} 2$ coated silicon wafer.

In Figure 9 a comparative study for different grating periods and phase-shifts is presented. The main conclusion is that for gratings with a lower number of periods the phase-shift band is sharper than in gratings with a higher number of periods. On the other hand, the reflection band of the grating presents a higher contrast compared to the rest of the spectrum for a higher number of periods.

In addition, the position of the phase-shift band in the middle of the FBG band indicates the precision in controlling the thickness of the defect (it was demonstrated in Visualization 1 that the position of the defect band is very sensitive to the defect layer thickness). 

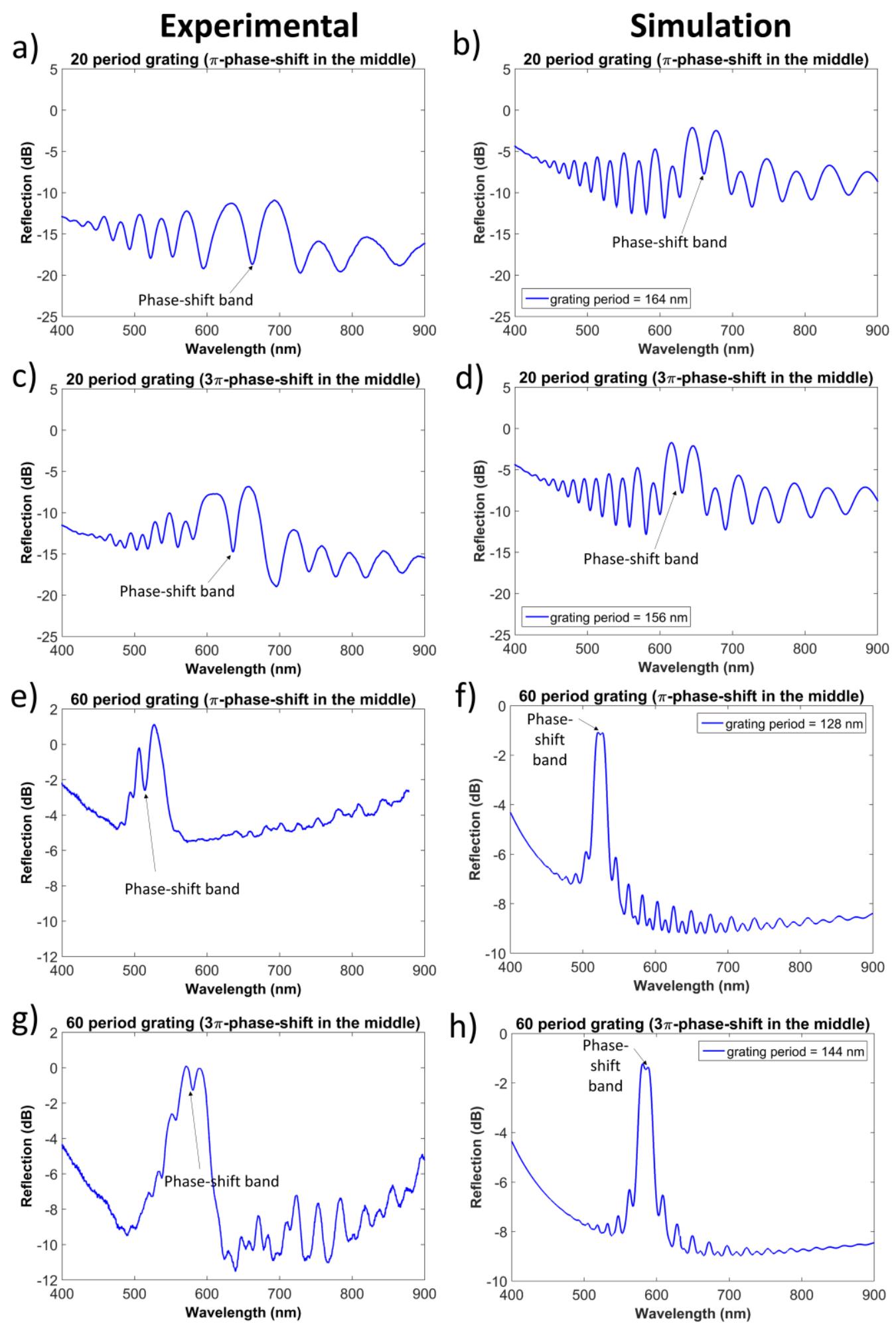

Fig. 9. Experimental constructions of phase-shifted gratings (a,c,e,g) and their respective simulations $(\mathbf{b}, \mathbf{d}, \mathbf{f}, \mathbf{h})$. 


\section{Humidity sensor}

The sensor composed of a 20 period grating with a $\pi$-phase-shift in the middle, whose spectrum is shown in Figure 9(a), was introduced in a climatic chamber (ACS CH 250 from Angelantoni Industries). Decreasing and increasing relative humidity ramps were induced (see the evolution in Figure 10(a) of the relative humidity measured by the climatic chamber humidity sensor), while temperature was kept constant at $23{ }^{\circ} \mathrm{C}$ during the experiment. In Fig. 10(b) the wavelength of the phase-shift band follows the evolution of the climate chamber sensor. The overall wavelength shift was $12 \mathrm{~nm}$, which indicates a sensitivity of $200 \mathrm{pm} / \%$ relative humidity. According to a recent review on humidity sensors [23], the sensitivity attained here improves by more than a factor of 5 the best values obtained with fiber Bragg gratings: $31 \mathrm{pm} / \%$ relative humidity with etched fiber Bragg gratings [24], and 35 pm/\% relative humidity with PMMAbased micro-structured polymer optical fiber Bragg gratings [25]. In addition, the results presented here improve those obtained in [26] with the same type of device but without a phaseshift. Moreover, the sensitivity could be increased if the hydrophilicity of the material deposited could be controlled [27]. 
a)
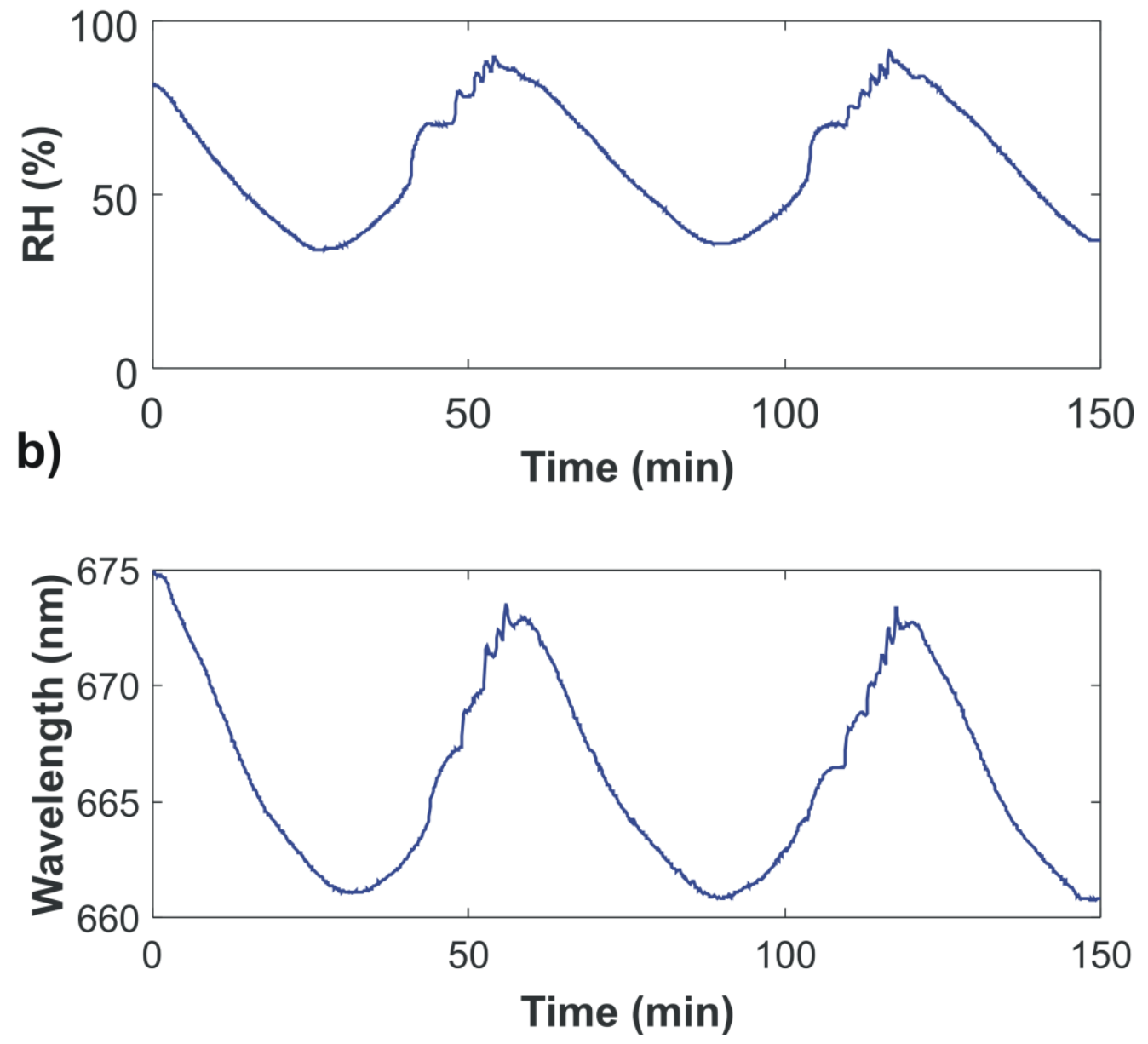

Fig. 10. Effect of the variation of humidity for a 20 period grating with a $\pi$-phase-shift in the middle: a) relative humidity; b) Wavelength of the phase-shift band.

In Figure 11 the same analysis was performed with steps of relative humidity: 30, 50, 70 and 90\%. The wavelength shift of the optical sensor in Figure 11(b) was again similar to the evolution of the relative humidity measured with the sensor of the climate chamber in Figure 11(a), whereas the overall wavelength shift was again $12 \mathrm{~nm}$. A small hysteresis of $0.7 \mathrm{~nm}$ was observed at humidity $50 \%$ by comparing the up and down phases, something that was not observed in the rest of cases analyzed. 
Finally, Figure 12 shows the temperature cross-sensitivity. The temperature range analyzed was $20^{\circ}$ to $30^{\circ} \mathrm{C}$ at constant humidity of $40 \%$ for a value of $30 \mathrm{pm} /{ }^{\circ} \mathrm{C}$. This cross sensitivity is equivalent to $0.15 \%$ relative humidity, which could be reduced by inducing an increase in the hydrophilicity of the $\mathrm{SnO}_{2}$ layers. The sensitivity would be higher and the influence of temperature would be reduced.

a)

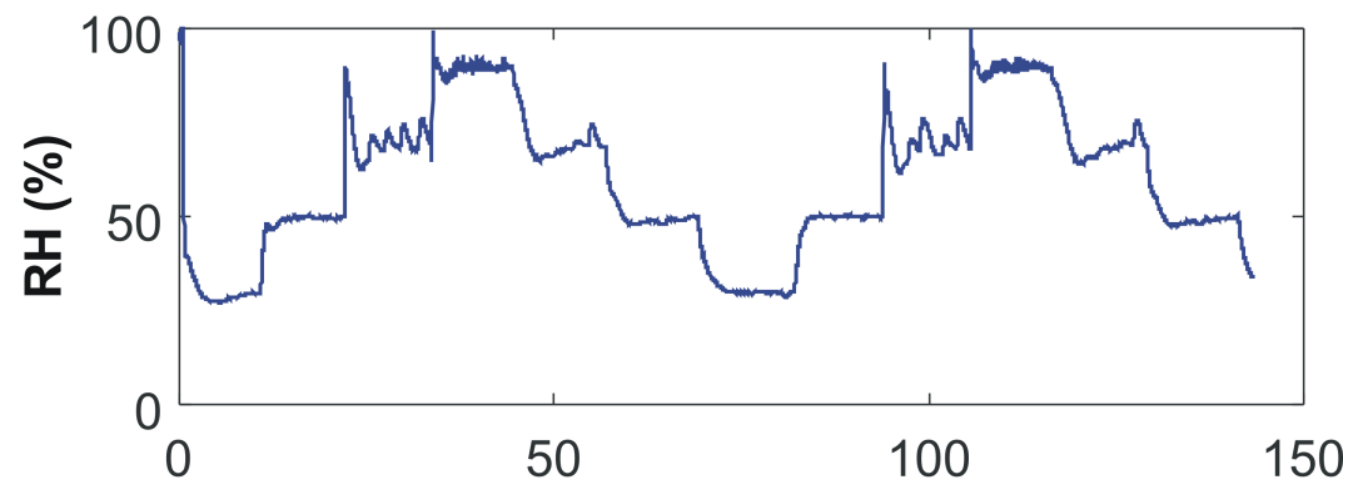

b)

Time (min)

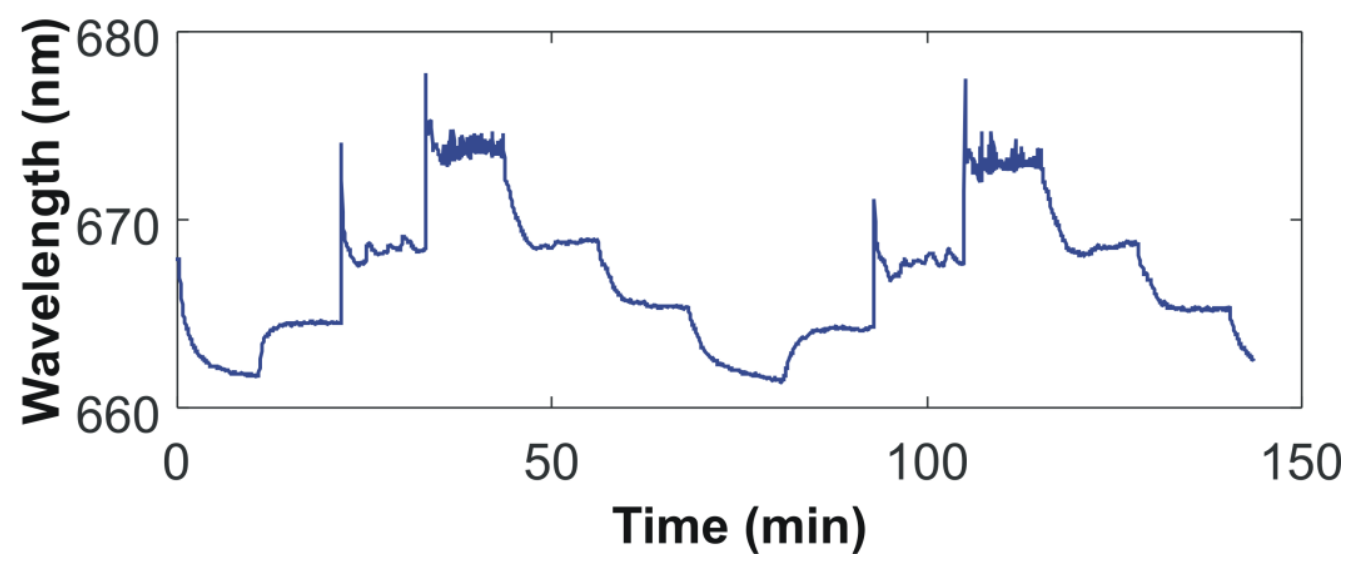

Fig. 11. Same as in Figure 10 but in steps: 30, 50, 70 and $90 \% \mathrm{RH}$ : a) relative humidity; b) Wavelength of the phase-shift band. 


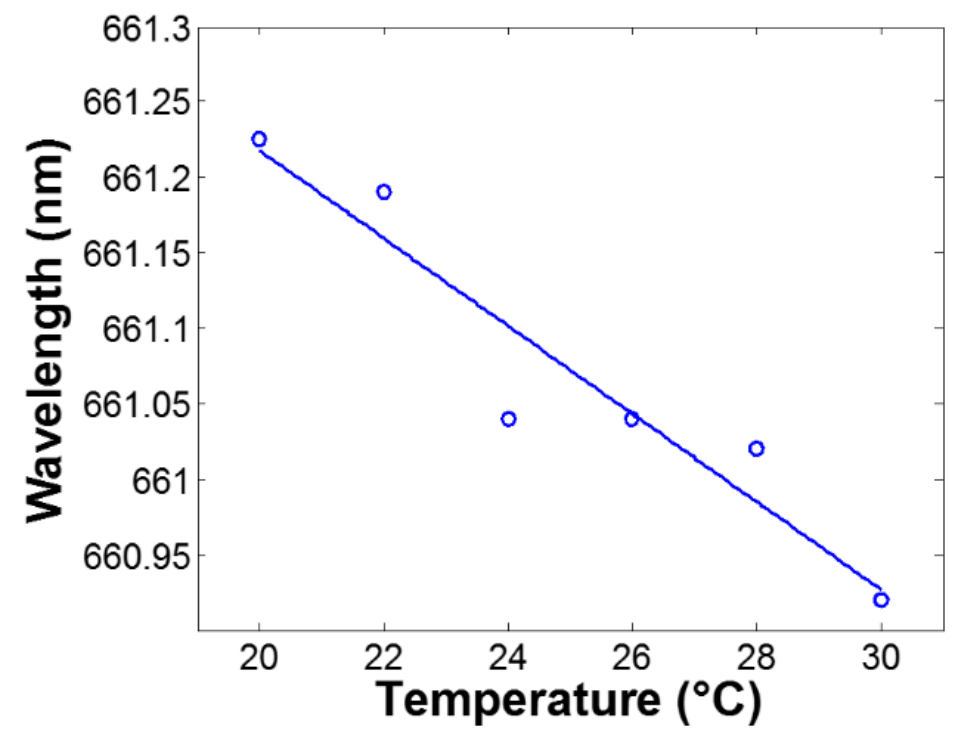

Figure 12. Temperature sensitivity of 20 period grating with $\pi$-phase-shift in the middle.

\section{CONCLUSIONS}

Phase-shifted gratings have been deposited on the end facet of a multimode optical fiber with a pulsed DC sputtering machine and a single target. The results obtained prove that it is possible to control the thickness of the defect (i.e. $\pi$-phase-shifted and $3 \pi$-phase-shifted gratings have been generated). The accuracy of the process allows positioning the phase-shift band in the middle of the reflection band. Moreover, it has been checked that the separation between the device and the target of the sputtering machine is responsible for the global grating thickness, which allows positioning the resonance band at a different position in the optical spectrum. The experimental results have been corroborated with simulations that provide a better understanding of the behavior of the devices under design.

As an application of the structure analyzed in this work, a humidity sensor has been successfully tested, which demonstrates the ability of these devices to be used as a sensing platform. The next 
step is to deposit a different material in the defect layer compared to the rest of the structure, which should expand more the field of application of this type of devices. As an example, the small size of the sensing head (the grating length is about $2 \mu \mathrm{m}$ ) suggests the possibility to apply this type of optical structure for detecting biological species that are present in small volumes. Another interesting characteristic of the phase-shifted Bragg gratings proposed here, compared to Bragg gratings without a phase-shift, is the possibility to optimize the full width at half maximum (FWHM), which is also interesting for sensing purposes. Here it has been observed that the dip created in the middle of the reflection band is narrower than the reflection band of the structure without phase-shift. Consequently, the path is open towards the optimization of the FWHM of this phase-shift band by selecting adequate parameters for the grating, such as the number of layers, the thickness, the real part of the refractive index or the imaginary part of the refractive index of the defect layer.

\section{ACKNOWLEDGMENTS}

This work was supported by the Spanish Agencia Estatal de Investigación (AEI) and Fondo Europeo de Desarrollo Regional (FEDER) (TEC2016-79367-C2-2-R,TEC2016-78047-R).

\section{REFERENCES}

1. V. Wilkens, C. Koch and W. Molkenstruck, "Frequency response of a fiber-optic dielectric multilayer hydrophone," 2000 IEEE Ultrasonics Symposium. Proceedings. An International Symposium (Cat. No.00CH37121), San Juan, 2000, pp. 1113-1116 vol. 2. 
2. F. J. Arregui, I. R. Matias, K. L. Cooper, and R. O. Claus, "Fabrication of microgratings on the ends of standard optical fibers by the electrostatic self-assembly monolayer process," Opt. Lett., vol. 26, no. 3, pp. 131-133, 2001. https://doi.org/10.1364/OL.26.000131.

3. M. F. Schubert, F. W. Mont, S. Chhajed, D. J. Poxson, J. K. Kim, and E. F. Schubert, "Design of multilayer antireflection coatings made from co-sputtered and low-refractive index materials by genetic algorithm," Opt. Express, vol. 16, no. 8, p. 5290 (9pages), 2008. https://doi.org/10.1364/OE.16.005290.

4. A. Szeghalmi, M. Helgert, R. Brunner, F. Heyroth, U. Gösele, and M. Knez, "Atomic layer deposition of $\mathrm{Al} 2 \mathrm{O} 3$ and $\mathrm{TiO} 2$ multilayers for applications as bandpass filters and antireflection coatings," Appl. Opt., vol. 48, no. 9, pp. 1727-1732, 2009. https://doi.org/10.1364/AO.48.001727.

5. I. Pavlichenko, A. T. Exner, M. Guehl, P. Lugli, G. Scarpa, and B. V. Lotsch, "Humidityenhanced thermally tunable TiO 2/SiO 2 Bragg stacks," J. Phys. Chem. C, vol. 116, no. 1, pp. 298-305, 2012. https://doi.org/10.1021/jp208733t.

6. Y. Tu, Y.-H. Qi, and S.-T. Tu, "Fabrication and thermal characteristics of multilayer metalcoated regenerated grating sensors for high-temperature sensing," Smart Mater. Struct., vol. 22, no. 7, p. 75026, 2013. https://doi.org/10.1088/0964-1726/22/7/075026.

7. C. Huang, D. Lee, J. Dai, W. Xie, and M. Yang, "Fabrication of high-temperature temperature sensor based on dielectric multilayer film on Sapphire fiber tip," Sens. Actuators A Phys., vol. 232, pp. 99-102, 2015. https://doi.org/10.1016/j.sna.2015.05.017.

8. S. G. Ilchenko, R. A. Lymarenko, and V. B. Taranenko, "Metal-Multilayer-Dielectric Structure for Enhancement of s- and p-Polarized Evanescent Waves," Nanoscale Res. Lett., vol. 11(1), p. 42, 2016. https://doi.org/10.1186/s11671-016-1274-3. 
9. M. Hála, R. Vernhes, O. Zabeida, J. E. Klemberg-Sapieha, and L. Martinu, "Reactive HiPIMS deposition of SiO2/Ta2O5 optical interference filters," J. Appl. Phys., vol. 116, pp. 213302, 2014. http://dx.doi.org/10.1063/1.4903285.

10. J. Ascorbe, J. M. Corres, I. Del Villar, F. J. Arregui and I. R. Matías, "Fabrication of Bragg Gratings on the End Facet of Standard Optical Fibers by Sputtering the Same Material," J. Lightwave Technol. vol. 35 pp. 212-219, 2017. http://dx.doi.org/10.1109/JLT.2016.2640021.

11. I. Del Villar, I. R. Matias, F. J. Arregui and R. O. Claus, "Analysis of one-dimensional photonic band gap structures with a liquid crystal defect towards development of fiber-optic tunable wavelength filters," Opt. Express vol. 11, pp. 430-436, 2003. https://doi.org/10.1364/OE.11.000430.

12. G. M. Nogueira, D. Banerjee, R. E. Cohen, M. F. Rubner, "Spray-layer-by-layer assembly can more rapidly produce optical-quality multistack heterostructures," Langmuir, vol. 27(12), pp. 7860-7867 2011. https://doi.org/10.1021/la200790g.

13. I. Safi, "Recent aspects concerning DC reactive magnetron sputtering of thin films: A review," Surf. Coat. Technol., vol. 127(2-3), pp. 203-219, 2000. https://doi.org/10.1016/S0257-8972(00)00566-1

14. C. Zhang, S. Liang, H. Zhu, W. Wang, "Tunable DFB lasers integrated with Ti thin film heaters fabricated with a simple procedure," Opt. Laser Technol., vol. 54, pp. 148-150, 2013. https://doi.org/10.1016/j.optlastec.2013.05.024.

15. M Yang, J Dai, C Zhou, D Jiang, "Optical fiber magnetic field sensors with TbDyFe magnetostrictive thin films as sensing materials," Opt. Express, vol. 17(23) pp. 20777-20782, 2009. https://doi.org/10.1364/OE.17.020777. 
16. J. Dai, M. Yang, X. Yu, H. Lu, "Optical hydrogen sensor based on etched fiber Bragg grating sputtered with Pd/Ag composite film" Opt. Fiber Technol., vol. 19(1), pp. 26-30, 2013. https://doi.org/10.1016/j.yofte.2012.09.006.

17. I. Del Villar, F. J. Arregui, C. R. Zamarreño, J. M. Corres, C. Bariáin, J. Goicoechea, C. Elosúa, M. Hernáez, P. J. Rivero, A. B. Socorro, A. Urrutia, P. Sánchez, P. Zubiate, D. López, N. De Acha, J. Ascorbe, I. R. Matías, “Optical sensors based on lossy mode resonances", Sens. Act. B, vol. 240, pp. 174-185, 2017. https://doi.org/10.1016/j.snb.2016.08.126.

18. T. Erdogan, "Fiber grating spectra," J. Lightwave Technol. vol. 15(8), pp. 1277-1294, 1997. https://doi.org/10.1109/50.618322.

19. D. Gatti, G. Galzerano, D. Janner, S. Longhi and P. Laporta, "Fiber strain sensor based on a $\pi$-phase-shifted Bragg grating and the Pound-Drever-Hall technique," Opt. Express vol. 16, pp. 1945-1950, 2008. https://doi.org/10.1364/OE.16.001945.

20. A. Rosenthal, D. Razansky, V. Ntziachristos, "High-sensitivity compact ultrasonic detector based on a pi-phase-shifted fiber Bragg grating," Opt. Lett. vol. 36(10), pp. 1833-1835, 2011. https://doi.org/10.1364/OL.36.001833.

21. W. Li, J. Yao, "A wideband frequency tunable optoelectronic oscillator incorporating a tunable microwave photonic filter based on phase-modulation to intensity-modulation conversion using a phase-shifted fiber bragg grating," IEEE Transactions on Microwave Theory and Techniques, vol. 60(6 PART 1), 6169961, pp. 1735-1742 2012. https://doi.org/10.1109/TMTT.2012.2189231.

22. I. H. Malitson, "Interspecimen comparison of the refractive index of fused silica," J. Opt. Soc. Am. vol. 55 (1965) 1205-1209. http:// doi.org/10.1364/JOSA.55.001205. 
23. J. Ascorbe, J. M. Corres, F. J. Arregui and I. R. Matias, "Recent Developments in Fiber Optics Humidity Sensors," Sensors, vol. 17, pp. 893, 2017.

24. G. Woyessa, K. Nielsen, A. Stefani, C. Markos, O. Bang, "Temperature insensitive hysteresis free highly sensitive polymer optical fiber Bragg grating humidity sensor," Opt. Express vol. 24, pp. 1206-1213, 2016. https:// doi.org/10.1364/OE.24.001206.

25. B. N. Shivananju, S. Yamdagni, R. Fazuldeen, A. K. S. Kumar, S. P. Nithin, M. M. Varma, S. Asokan, "Highly Sensitive Carbon Nanotubes Coated Etched Fiber Bragg Grating Sensor for Humidity Sensing,” IEEE Sens. J. vol. 14, pp. 2615-2619, 2014. https:// doi.org/10.1109/JSEN.2014.2312353.

26. J. Ascorbe, J. M. Corres, F. J. Arregui and I. R. Matias, "Humidity sensor based on bragg gratings developed on the end facet of an optical fiber by sputtering of one single material," Sensors, vol. 17(5), pp. 991 (2017).

27. M. Miyauchi, A. Nakajima, T. Watanabe, "Photocatalysis and Photoinduced Hydrophilicity of Various Metal Oxide Thin Films," Chem. Mater. vol. 14, pp. 2812-2816 (2002). https:// doi.org/10.1021/cm020076p. 